\title{
Mappings preserving locations of movable poles: a new extension of the truncation method to ordinary differential equations
}

\author{
Pilar R. Gordoa, Nalini Joshi†and Andrew Pickering ${ }^{\ddagger}$ \\ Department of Pure Mathematics \\ University of Adelaide \\ Adelaide Australia 5005
}

November 15, 2018

\begin{abstract}
The truncation method is a collective name for techniques that arise from truncating a Laurent series expansion (with leading term) of generic solutions of nonlinear partial differential equations (PDEs). Despite its utility in finding Bäcklund transformations and other remarkable properties of integrable PDEs, it has not been generally extended to ordinary differential equations (ODEs). Here we give a new general method that provides such an extension and show how to apply it to the classical nonlinear ODEs called the Painlevé equations. Our main new idea is to consider mappings that preserve the locations of a natural subset of the movable poles admitted by the equation. In this way we are able to recover all known fundamental Bäcklund transformations for the equations considered. We are also able to derive Bäcklund transformations onto other ODEs in the Painlevé classification.
\end{abstract}

*permanent address: Facultad de Ciencias, Edificio de Fisica, Universidad de Salamanca, 37008 Salamanca, Spain, prg@sonia.usal.es

${ }^{\dagger}$ njoshi@maths. adelaide.edu. au

${ }^{\ddagger}$ current address: Facultad de Ciencias, Edificio de Fisica, Universidad de Salamanca, 37008 Salamanca, Spain, andrew@sonia.usal.es 


\section{Introduction}

Integrable differential equations are those that are solvable (for a large space of initial data) through an associated linear problem[1]. It is conjectured that the solutions of all such equations possess a characteristic complex singularity structure [2]. In particular, there is widespread evidence that all movable singularities of all solutions are poles [3, [1]. This is commonly referred to as the Painlevé property. Extensions of this definition can be found for example in 5$]$.

This property has been used as a starting point for deducing other remarkable properties of integrable partial differential equations (PDEs). The basic idea, first proposed by Weiss [6] (see also [7]), is to truncate a Laurent expansion of a generic solution near a movable pole. For example, the Korteweg-deVries (KdV) equation

$$
u_{t}+6 u u_{x}+u_{x x x}=0, \quad u=u(x, t)
$$

admits locally convergent Laurent series of the form [8, 9]

$$
u=\frac{-2 \Phi_{x}^{2}}{\Phi^{2}}+\frac{2 \Phi_{x x}}{\Phi}+\sum_{n=0}^{\infty} u_{n}(x, t) \Phi(x, t)^{n},
$$

as solutions in a neighbourhood of any analytic, noncharacteristic variety given by $\Phi(x, t)=0$. (Note that noncharacteristic here implies $\Phi_{x} \neq 0$.) The "truncation" of this series is

$$
u=\frac{-2 \Phi_{x}^{2}}{\Phi^{2}}+\frac{2 \Phi_{x x}}{\Phi}+g(x, t)
$$

where $g(x, t)$ (often called the "constant-level" term) is analytic near $\Phi=0$. Asking that this expression be a solution of the KdV equation, order by order in $\Phi$, requires that $g$ must be a solution of the KdV equation, and also that $\Phi$ satisfies an equation, often referred to as the "singular manifold equation." Weiss showed how it is then possible to deduce the well known linear problem and Darboux transformation for the KdV equation. These then yield the Bäcklund transformation for the KdV equation. Such ideas have led to a procedure called the "truncation method" that has been successfully extended [10, 11, 12, 13, 14, 15] to many PDEs.

However, no such general procedure exists for ODEs. The PDE-truncation procedure relies on setting coefficients of different powers of $\Phi$ to zero in the image equation obtained by substituting the truncation. This poses a difficulty for ODEs. In each case for which this has been tried for an ODE 
[16, 17, 18, 19], the results have been found to be very restricted. For example, only special transformations or special solutions have been found via this procedure. In particular, no general, parameter-dependent Bäcklund transformation has been found by a truncation method.

Recently, Clarkson, Joshi and Pickering [20] have shown how this difficulty can be overcome for the second Painlevé $\left(\mathrm{P}_{\mathrm{II}}\right)$ hierarchy. The main idea here was to use the truncation and the image equation (obtained by substituting the truncation) to eliminate $\Phi$, instead of separating powers of $\Phi$. The result is a Bäcklund transformation for the hierarchy. This approach has also been extended to the reductions of the modified Sawada-Kotera/KaupKupershmidt (mSK/mKK) hierarchy [21].

Our purpose here is to give a universal truncation-type method for ODEs that is based on singularity analysis. The above applications (to the $\mathrm{P}_{\mathrm{II}}$ and reduced $\mathrm{mSK} / \mathrm{mKK}$ hierarchies) can be recast in this framework. The main new idea is to consider truncation as a mapping that preserves the locations of a natural subset of movable singularities.

An example is given by the second Painlevé equation

$$
y^{\prime \prime}=2 y^{3}+x y+\alpha, \quad y=y(x),
$$

whose general solution possesses two families of movable poles [22]. (These are often referred to in the literature as two "branches" of a Painlevé expansion.) Near a movable singularity $x=x_{0}$, say, $y(x)$ has a convergent Laurent expansion

$$
y=\frac{ \pm 1}{\left(x-x_{0}\right)}+h(x)\left(x-x_{0}\right),
$$

where $h(x)$ is locally analytic. Clearly, the set of poles of $y(x)$ naturally separates into two subsets, identified by the sign of the coefficient of the leading-order pole.

Each of the Painlevé equations, except the first, has generic solution $y(x)$ that possesses pairs of simple movable poles with coefficients of opposite sign. Therefore, the set of all movable poles of a solution $y(x)$ decomposes into the union of two nonintersecting subsets $\mathcal{P}_{+}$and $\mathcal{P}_{-}$. By $\mathcal{P}_{+}$we mean the set of poles with positive choice of coefficient and $\mathcal{P}_{-}$is that with the negative choice.

In the following section, a generic solution $y(x)$ of a Painlevé equation will be transformed to a solution $Q(x)$ of the same equation but with possibly different parameters as

$$
y(x)=\rho(x)+Q(x) .
$$

We construct the transformation by demanding that $\rho(x)$ have poles exactly at the elements of $\mathcal{P}_{+}$and $Q(x)$ have them at $\mathcal{P}_{-}$(or vice-versa). When 
$Q(x)$ satisfies the same equation (albeit with different parameters), we find Bäcklund transformations (BTs), through a procedure that relies only on singularity analysis of the transformed equation.

The method is applied to $\mathrm{P}_{\mathrm{II}}$ and $\mathrm{P}_{\mathrm{IV}}$ in this paper. In section 2, we give details for $\mathrm{P}_{\mathrm{II}}$ and indicate the major differences for $\mathrm{P}_{\mathrm{IV}}$. This method also leads to related ODEs and Bäcklund transformations between these and the equation under consideration; these are considered in Section 3, again for $\mathrm{P}_{\mathrm{II}}$ and $\mathrm{P}_{\mathrm{IV}}$. In section 4 , we show how to carry out the analogue of the so called double singular manifold method, i.e. one which considers two singularities of the solution simultaneously. Extensions of these techniques to other Painlevé equations are considered in a second paper [23].

\section{Truncation for $\mathrm{P}_{\mathrm{II}}$ and $\mathrm{P}_{\mathrm{IV}}$}

In this section we show how to carry out a mapping that preserves "half" the movable poles of the solution of a Painlevé equation and how this can lead to a Bäcklund transformation for that equation.

We recall the equations $\mathrm{P}_{\mathrm{II}}$ and $\mathrm{P}_{\mathrm{IV}}$ here for reference later.

$$
\begin{aligned}
& y^{\prime \prime}=2 y^{3}+x y+\alpha \\
& y^{\prime \prime}=\frac{y^{\prime 2}}{2 y}+\frac{3 y^{3}}{2}+4 x y^{2}+2\left(x^{2}-\alpha\right) y-\frac{\gamma^{2}}{2 y}
\end{aligned}
$$

(Note the slightly unusual renaming of the second parameter in $\mathrm{P}_{\mathrm{IV}}$ that differs from convention.) We take $Q(x)$ to satisfy the same equation as $y(x)$ but with possibly different parameters indicated by roman letters replacing the corresponding greek ones. (So e.g. $\alpha \mapsto a$.)

\section{$2.1 \quad \mathrm{P}_{\text {II }}$}

Substitution of Eq (1.5) into $\mathrm{P}_{\mathrm{II}}$ gives

$$
\rho^{\prime \prime}-2 \rho^{3}-6 \rho^{2} Q-6 \rho Q^{2}-x \rho-\alpha+a=0 .
$$

The dominant terms of this equation near a pole of $\rho$ are

$$
\rho^{\prime \prime} \approx 2 \rho^{3} \Rightarrow \rho^{\prime 2} \approx \rho^{4}
$$

Taking the positive square root (i.e. taking $\rho$ to have a pole in $\mathcal{P}_{-}$), we write

$$
\rho^{\prime}(x)=: \rho(x)^{2}+\sigma(x) \rho(x),
$$


where $\sigma$ is to be found. Using this to replace $\rho^{\prime}$ in the equation (2.8), we get

$$
(3 \sigma(x)-6 Q(x)) \rho(x)^{2}+\left(-x+\sigma(x)^{2}+\sigma^{\prime}(x)-6 Q(x)^{2}\right) \rho(x)-\alpha+a=0 .
$$

Consider the dominant terms of this equation near a pole of $\rho$. Since $Q(x)$ is regular at such points, we get to leading-order

$$
\sigma(x) \approx 2 Q(x) \Rightarrow \sigma(x)=: 2 Q(x)+\frac{\tau(x)}{\rho(x)}
$$

Now Eq (2.8) becomes

$$
\left(-x+2 Q^{\prime}(x)-2 Q(x)^{2}+2 \tau(x)\right) \rho(x)+2 \tau(x) Q(x)+\tau^{\prime}(x)+a-\alpha=0,
$$

from which we have

$$
\rho(x)=-\frac{2 \tau(x) Q(x)+\tau^{\prime}(x)+a-\alpha}{-x+2 Q^{\prime}(x)-2 Q(x)^{2}+2 \tau(x)} .
$$

However, this must be compatible with the Riccati equation (2.9) for $\rho(x)$. Substituting this expression for $\rho(x)$ into (2.9) yields a compatibility condition that contains $Q, Q^{\prime}$ and $Q^{\prime \prime}$. We use the equation $\mathrm{P}_{\text {II }}$ satisfied by $Q(x)$ to eliminate $Q^{\prime \prime}$. The result is a polynomial equation in $Q$ and $Q^{\prime}$, with coefficients involving $\tau, \tau^{\prime}$ and $\tau^{\prime \prime}$, given by

$$
\begin{aligned}
0= & -4 \tau(x) Q(x)^{4}+4 \tau^{\prime}(x) Q(x)^{3} \\
& +\left(12 \tau(x)^{2}+12 \tau(x) Q^{\prime}(x)+2 \tau^{\prime \prime}(x)-4 x \tau(x)\right) Q(x)^{2} \\
& +\left(-2 \tau(x)+2 x \tau^{\prime}(x)-4 Q^{\prime}(x) \tau^{\prime}(x)+4 a \tau(x)\right) Q(x) \\
& -8 \tau(x)\left(Q^{\prime}(x)\right)^{2} \\
& +\left(-2 \tau^{\prime \prime}(x)+6 x \tau(x)-12 \tau(x)^{2}\right) Q^{\prime}(x) \\
& -x^{2} \tau(x)+a^{2}-\alpha^{2}+\alpha-a+2 a \tau^{\prime}(x) \\
& -\tau^{\prime}(x)+\left(\tau^{\prime}(x)\right)^{2} \\
& -2 \tau^{\prime \prime}(x) \tau(x)-4 \tau(x)^{3}+x \tau^{\prime \prime}(x)+4 x \tau(x)^{2}
\end{aligned}
$$

Since the solution space of $\mathrm{P}_{\mathrm{II}}$ depends on two arbitrary parameters given by $Q, Q^{\prime}$ at a (regular) point, we can demand that this equation be satisfied identically in these two variables. Since $\rho$ is a functional of $Q$ (or equivalently $y), \tau$ is also. The simplest solutions of $\mathrm{Eq}(2.10)$ are those that are polynomial in $Q$ and $Q^{\prime}$. Inspection of $\mathrm{Eq}(2.10)$ shows that the simplest solution independent of both $Q$ and $Q^{\prime}$ is $\tau(x) \equiv 0$ (because of the presence of the monomial $\left.4 \tau(x) Q(x)^{4}\right)$ under the constraint

$$
a^{2}-a=\alpha^{2}-\alpha \Rightarrow a=\alpha \text { or } a=-\alpha+1 \text {. }
$$


Then, the above solution for $\rho$ becomes

$$
\rho(x)=\frac{a-\alpha}{x-2 Q^{\prime}(x)+2 Q(x)^{2}} .
$$

Thus in addition to the identity $y(x)=Q(x)$ we obtain the well known BT for $\mathrm{P}_{\mathrm{II}}$ [24].

We have also considered other possible solutions for $\tau$, e.g. those that only depend polynomially on $Q$ but not $Q^{\prime}$. The monomial $-2 \tau^{\prime \prime}(x) Q^{\prime}(x)$ in Eq (2.10) shows that it can be at most linear in $Q$. Other monomials in the resulting equation then lead again to $\tau(x) \equiv 0$. (For other equations, a possible dependence of $\tau$ on $Q$ can give more general results [23].)

If in $\mathrm{Eq}(2.9)$ we had taken the negative root, i.e.

$$
\rho^{\prime}(x)=-\rho(x)^{2}+\sigma(x) \rho(x)
$$

the above procedure would have yielded

$$
\rho(x)=\frac{a-\alpha}{x+2 Q^{\prime}(x)+2 Q(x)^{2}}, \quad a=-\alpha-1,
$$

the alternative form of the BT for $\mathrm{P}_{\mathrm{II}}$. This could also have been found by using the discrete symmetry of $\mathrm{P}_{\text {II }}$ under $y \mapsto-y, \alpha \mapsto-\alpha$ in combination with Eqn (2.11). It should be noted, however, that both BTs are needed in order to iterate to find sequences of special integrals and rational solutions.

\section{$2.2 \quad \mathrm{P}_{\mathrm{IV}}$}

Here we describe major differences in applying the above procedure to $\mathrm{P}_{\mathrm{IV}}$. The first difference lies in the dominant terms of the transformed equation. These are

$$
\rho^{\prime \prime} \rho-\frac{\rho^{\prime 2}}{2} \approx \frac{3 \rho^{4}}{2}
$$

After using the integrating factor $\rho^{\prime} \rho^{-2}$, we can integrate to get

$$
\frac{\rho^{\prime 2}}{\rho} \approx \rho^{3} \Rightarrow \rho^{\prime} \approx \pm \rho^{2}
$$

Consider first the case with minus sign on the right, i.e.

$$
\rho^{\prime}=-\rho^{2}+\sigma \rho
$$

Then we find (following dominant balances again) that

$$
\sigma(x)=-2 x-2 Q(x)+\frac{\tau(x)}{\rho(x)}
$$


Using this in the transformed equation leads to a quadratic equation for $\rho$ :

$$
\begin{aligned}
& \left(-2 x Q(x)-\tau(x)-Q(x)^{2}-2+2 \alpha-Q^{\prime}(x)\right) \rho(x)^{2}+ \\
& \left(4 \alpha Q(x)-\frac{1}{2} Q(x)^{3}-2 Q(x) a-\frac{1}{2} \frac{c^{2}}{Q(x)}+\frac{1}{2} \frac{Q^{\prime}(x)^{2}}{Q(x)}\right. \\
& \left.+\tau^{\prime}(x)+2 x^{2} Q(x)-2 \tau(x) Q(x)-2 Q(x)+2 Q^{\prime}(x) x\right) \rho(x) \\
& -2 Q(x) \tau(x) x-2 Q(x)^{2} a-\frac{1}{2} \tau(x)^{2}+\frac{1}{2} \gamma^{2}-Q^{\prime}(x) \tau(x) \\
& +Q(x) \tau^{\prime}(x)+2 \alpha Q(x)^{2}-2 \tau(x) Q(x)^{2}-\frac{1}{2} c^{2}=0 .
\end{aligned}
$$

That we obtain a quadratic equation in $\rho$ is a second difference between our results for $\mathrm{P}_{\mathrm{II}}$ and $\mathrm{P}_{\mathrm{IV}}$. In general (beginning with a polynomial ODE that gives a Riccati equation for $\rho$ ) we could obtain at this stage a polynomial of higher degree in $\rho$.

This quadratic equation must be compatible with the differential equation

$$
\rho^{\prime}(x)=-\rho(x)^{2}-(2 x+2 Q(x)) \rho(x)+\tau(x),
$$

for $\rho(x)$. Since equation (2.12) is quadratic in $\rho$, it is worth noting how we obtain a unique $\rho$ to check the compatibility condition. Suppose we differentiate Eqn (2.12) w.r.t. $x$ and use Eqn (2.13) to replace $\rho^{\prime}$. Then we obtain a cubic equation in $\rho$. The third degree term can be eliminated by using Eqn (2.12) multiplied by $\rho$. This yields another quadratic equation for $\rho$. Eliminating the second-degree term by using Eqn (2.12) again, we get a linear equation for $\rho$, which we solve. The result is substituted into Eqn (2.13) to obtain the compatibility condition we investigate.

This equation can be analysed to find $\tau$ and any conditions on the parameters. We assume here that $\tau$ is independent of both $Q$ and $Q^{\prime}$; the final results are the same if we allow $\tau$ to depend on $Q$. The coefficient of $Q^{13}$ gives

$$
\tau(x)=\frac{2}{3}(\alpha-a)
$$

Substitution of this into the compatibility condition yields

$$
c^{2}=\gamma^{2}+\frac{4}{3}\left(\alpha^{2}-a^{2}-2 \alpha+2 a\right) .
$$

With this choice of $c^{2}$, the remaining terms in the compatibility condition factor to give

$$
(\alpha-a)(2 \alpha-6-3 \gamma+4 a)(2 \alpha-6+3 \gamma+4 a)=0 .
$$


Thus in addition to the identity $y(x)=Q(x)$ we obtain the two nontrivial BTs

$$
\begin{gathered}
\rho(x)=\frac{( \pm \gamma-2+2 \alpha) Q(x)}{Q^{\prime}(x)+Q(x)^{2}+2 x Q(x) \pm \gamma / 2+1-\alpha} \\
a=-\frac{1}{4}(2 \alpha-6 \pm 3 \gamma) \\
c^{2}=(\alpha \mp \gamma / 2-1)^{2}
\end{gathered}
$$

where the choice of sign of $\gamma$ (and $c$ ) arises because of the way we have written the second parameter in $\mathrm{P}_{\mathrm{IV}}$. This is the BT labelled by "dagger" $\left(y^{\dagger}\right)$ in 25.

The alternative choice of sign in the dominant balance of terms in $\rho$ leads to the Riccati equation

$$
\rho^{\prime}(x)=\rho(x)^{2}+\sigma(x) \rho(x)
$$

with

$$
\sigma(x)=2 Q(x)+2 x+\tau(x) / \rho(x)
$$

where

$$
\tau(x)=\frac{2}{3}(a-\alpha)
$$

This leads to the two BTs

$$
\begin{gathered}
\rho(x)=\frac{( \pm \gamma-2-2 \alpha) Q(x)}{Q^{\prime}(x)-Q(x)^{2}-2 x Q(x) \pm \gamma / 2+1+\alpha} \\
a=-\frac{1}{4}(2 \alpha+6 \mp 3 \gamma) \\
c^{2}=(\alpha \pm \gamma / 2+1)^{2}
\end{gathered}
$$

This is the BT labelled by "double dagger" $\left(y^{\ddagger}\right)$ in [25].

It is interesting to note that all the known BTs [25] of $\mathrm{P}_{\mathrm{IV}}$ can be expressed in terms of only two fundamental BTs. In [25], these two BTs are labelled by "hat" $(\hat{y})$ and "tilde" $(\widetilde{y})$. In the next section, we show that our derivation of the dagger and double dagger BTs also gives rise to these two fundamental BTs. 


\section{Related BTs: the ODEs satisfied by $\rho(x)$}

The results of the previous Section were obtained by searching for autoBäcklund transformations. That is, we looked for transformations between two copies of the same equation distinguished by possibly different parameter values. In this Section, we consider the ODE satisfied by the function $\rho(x)$ in (1.5), and deduce the Bäcklund transformation between this ODE and the Painlevé equation.

\section{$3.1 \quad \mathrm{P}_{\mathrm{II}}$}

In constructing our first auto-BT for $\mathrm{P}_{\mathrm{II}}$ (see the previous Section), we obtained a solution $\rho$ of the Riccati equation

$$
\rho^{\prime}(x)=\rho^{2}+2 Q(x) \rho(x)
$$

given by

$$
\rho(x)=\frac{1-2 \alpha}{2 Q(x)^{2}-2 Q^{\prime}(x)+x},
$$

where $Q(x)$ satisfies $\mathrm{P}_{\text {II }}$ with parameter $a=-\alpha+1$. Now we eliminate $Q(x)$ from the above equations, to find a second order ODE satisfied by $\rho(x)$, together with Bäcklund transformations to $\mathrm{P}_{\mathrm{II}}$ (in both $y(x)$ and $Q(x)$ ).

Eliminating $Q(x)$ between the above two equations gives

$$
\rho^{\prime \prime}(x)=\frac{3}{2} \frac{\rho^{\prime}(x)^{2}}{\rho(x)}+\frac{1}{2} \rho(x)^{3}+x \rho(x)-(1-2 \alpha)
$$

which under $\rho(x)=1 / s(x)$ becomes

$$
s^{\prime \prime}(x)=\frac{1}{2} \frac{s^{\prime}(x)^{2}}{s(x)}+(1-2 \alpha) s(x)^{2}-x s(x)-\frac{1}{2 s(x)}
$$

which is $\mathrm{P}_{\text {xxxiv }}$ (i.e. the thirty-fourth equation in the classification results presented in Chapter 14 of [26]). Using this change of variables, and the expression for $Q$ in terms of $\rho$, the BT (1.5) becomes

$$
y(x)=\frac{1-s^{\prime}(x)}{2 s(x)} .
$$

Therefore, we recover the well-known mapping between $\mathrm{P}_{\mathrm{XxxIv}}$ and $\mathrm{P}_{\mathrm{II}}$. We also have, of course, a Bäcklund transformation from $\mathrm{P}_{\text {xxxIV }}$ to $\mathrm{P}_{\mathrm{II}}$ in $Q(x)$. If we had started with the second Bäcklund transformation for $\mathrm{P}_{\mathrm{II}}$, we again find (after a simple change of variables) the mapping to $\mathrm{P}_{\mathrm{xxxIV}}$. 


\section{$3.2 \quad \mathrm{P}_{\mathrm{IV}}$}

We now consider the ODE satisfied by $\rho(x)$ in our construction of Bäcklund transformations for $\mathrm{P}_{\mathrm{IV}}$. Recall from Equations (2.13), (2.14) and (2.16), that $\rho(x)$ satisfies the Riccati equation

$$
\rho^{\prime}(x)=-\rho^{2}-2(Q(x)+x) \rho(x)+(\alpha-1 \pm \gamma / 2),
$$

for the dagger transformation. Elimination of $Q(x)$ between this and 2.15) gives, after the substitution $\rho(x)= \pm C / s(x)$, the ODE

$$
s^{\prime \prime}(x)=\frac{1}{2} \frac{s^{\prime}(x)^{2}}{s(x)}+\frac{3}{2} s(x)^{3}+4 x s(x)^{2}+2\left(x^{2}-A\right) s(x)-\frac{C^{2}}{2 s(x)}
$$

where

$$
\begin{aligned}
A & =-\frac{1}{2}-\frac{1}{2} \alpha \pm \frac{3}{4} \gamma \\
\pm C & =1-\alpha \mp \frac{1}{2} \gamma .
\end{aligned}
$$

This is another copy of the same equation, $\mathrm{P}_{\mathrm{IV}}$. The corresponding autoBäcklund transformation is obtained from (1.5) after eliminating $Q(x)$ by using Equation (3.18). The result is

$$
y(x)=\frac{s^{\prime}(x)-s(x)^{2}-2 x s(x)+(1-\alpha \mp \gamma / 2)}{2 s(x)} .
$$

This is the BT labelled by "tilde" $(\widetilde{y})$ in 25].

On the other hand, Equation (2.15) gives

$$
s(x)=-\frac{Q^{\prime}(x)+Q(x)^{2}+2 x Q(x)+(1+A \pm C / 2)}{2 Q(x)}
$$

where by replacing $\alpha, \gamma$ in terms of $A, C$ above, we get the parameters $a, c$ of the version of $\mathrm{P}_{\mathrm{IV}}$ satisfied by $Q(x)$ as

$$
\begin{aligned}
a & =\frac{1}{2}-\frac{1}{2} A \pm \frac{3}{4} C \\
c^{2} & =\frac{1}{4}(2+2 A \pm C)^{2} .
\end{aligned}
$$

Hence we get another BT for $\mathrm{P}_{\mathrm{IV}}$. This is the BT labelled by "hat" $(\hat{y})$ in [25]. That is, we have used our derivation of $y^{\dagger}$ to deduce both $\widetilde{y}$ and $\hat{y}$.

The BTs $\widetilde{y}$ and $\hat{y}$ are fundamental BTs in the sense that all known BTs for $\mathrm{P}_{\mathrm{IV}}$ can be expressed in terms of these two. (See [25].) Our approach 
shows that $y^{\dagger}$ which maps $Q(x)$ to $y(x)$ can be considered, with appropriate choices of signs, as the composition $\widetilde{y} \circ \hat{y}$.

Now consider our derivation of the double dagger BT in Section 2.2. Following the same procedure of elimination of $Q(x)$, we find (with the substitution $\rho=\mp C / s(x)$ ) the same $\mathrm{P}_{\mathrm{IV}}$ i.e. Equation (3.19), but with $A$ and $C$ given now by

$$
\begin{aligned}
A & =\frac{1}{2}-\frac{1}{2} \alpha \mp \frac{3}{4} \gamma \\
\pm C & =1+\alpha \mp \frac{1}{2} \gamma .
\end{aligned}
$$

The corresponding BT for $\mathrm{P}_{\mathrm{IV}}$ is

$$
y(x)=-\frac{s^{\prime}(x)+s(x)^{2}+2 x s(x)+(1+\alpha \mp \gamma / 2)}{2 s(x)} .
$$

This is $\hat{y}$. Elimination of $y(x)$ instead gives

$$
s(x)=\frac{Q^{\prime}(x)-Q(x)^{2}-2 x Q(x)+(1-A \pm C / 2)}{2 Q(x)}
$$

where

$$
\begin{aligned}
a & =-\frac{1}{2}-\frac{1}{2} A \mp \frac{3}{4} C \\
c^{2} & =\frac{1}{4}(2-2 A \pm C)^{2},
\end{aligned}
$$

which is $\widetilde{y}$. Thus our derivation of $y^{\ddagger}$ also allows us to obtain the fundamental BTs $\widetilde{y}$ and $\hat{y}$. Our approach then shows that, for suitable choices of signs, $y^{\ddagger}$ can be expressed in terms of $\widetilde{y}$ and $\hat{y}$ as the composition $\hat{y} \circ \widetilde{y}$ [25].

\section{The double-singularity approach}

In Sections 2 and 3, we assumed that $\rho$ inherited half the poles of the Painlevé transcendent $y(x)$. Now we consider the possibility that both families of movable poles of $y(x)$ are inherited by specified functions called respectively $\rho_{1}$ and $\rho_{2}$ in the transformation. That is, we rewrite $y$ as

$$
y(x)=\rho_{1}(x)-\rho_{2}(x)+Y(x),
$$

where we assume that $Y(x)$ satisfies $\mathrm{P}_{\mathrm{II}}$, or respectively $\mathrm{P}_{\mathrm{IV}}$, with different parameters ( $a$, or respectively $a$ and $c$ ). 
As before, dominant terms of $\mathrm{P}_{\mathrm{II}}$ or $\mathrm{P}_{\mathrm{IV}}$ lead to Riccati equations for $\rho_{i}$ to leading-order near a movable pole. The dominant terms in each equation are as before. However, the lower-order terms cannot be uniquely determined by dominant balances. For simplicity, we choose the Riccati equation in $\rho_{1}$, to be linear in $\rho_{2}$ and vice versa.

Consider the Riccati equations for $\rho_{i}$ to have the form

$$
\begin{gathered}
\rho_{1}{ }^{\prime}(x)=\rho_{1}(x)^{2}+A_{1}(x) \rho_{1}(x) \rho_{2}(x)+B_{1}(x) \rho_{1}(x) \\
\quad+C_{1}(x) \rho_{2}(x)+\tau_{1}(x) \\
\rho_{2}{ }^{\prime}(x)=\quad \rho_{2}(x)^{2}+A_{2}(x) \rho_{1}(x) \rho_{2}(x)+B_{2}(x) \rho_{1}(x) \\
\quad+C_{2}(x) \rho_{2}(x)+\tau_{2}(x)
\end{gathered}
$$

In this case, where we take the same sign against $\rho_{i}^{2}$ in each Riccati equation, this approach is analagous to the double singular manifold method. However, we show at the end of each subsection below that we can also obtain nontrivial results by taking opposite signs in the above Riccati equations.

\section{$4.1 \quad \mathrm{P}_{\mathrm{II}}$}

We transform $\mathrm{P}_{\mathrm{II}}$ by using the relation (4.20) and equations (4.21), (4.22). Then we get

$$
\begin{aligned}
-\alpha & +a-C_{2}(x) \tau_{2}(x)+\tau_{1}{ }^{\prime}(x)+B_{1}(x) \tau_{1}(x)-B_{2}(x) \tau_{1}(x) \\
+ & C_{1}(x) \tau_{2}(x)-\tau_{2}{ }^{\prime}(x) \\
+ & \left(3 B_{1}(x)-B_{2}(x)-6 Y(x)-A_{2}(x) B_{2}(x)+A_{1}(x) B_{2}(x)\right) \rho_{1}(x)^{2} \\
+ & \left(B_{1}{ }^{\prime}(x)-B_{2}(x) B_{1}(x)+C_{1}(x) B_{2}(x)-B_{2}{ }^{\prime}(x)\right. \\
& -6 Y(x)^{2}+A_{1}(x) \tau_{2}(x)+B_{1}(x)^{2}-C_{2}(x) B_{2}(x)+2 \tau_{1}(x)-x \\
& \left.-A_{2}(x) \tau_{2}(x)\right) \rho_{1}(x)+\left(C_{1}(x) C_{2}(x)+A_{1}(x) \tau_{1}(x)+6 Y(x)^{2}\right. \\
& +B_{1}(x) C_{1}(x)-C_{2}(x)^{2}+x+C_{1}{ }^{\prime}(x)-C_{2}{ }^{\prime}(x)-2 \tau_{2}(x) \\
& \left.-C_{1}(x) B_{2}(x)-A_{2}(x) \tau_{1}(x)\right) \rho_{2}(x) \\
+ & \left(C_{1}(x)-6 Y(x)-3 C_{2}(x)-C_{1}(x) A_{2}(x)+A_{1}(x) C_{1}(x)\right) \rho_{2}(x)^{2} \\
+ & \left(-A_{2}(x)+A_{1}(x) A_{2}(x)+3 A_{1}(x)+6-A_{2}(x)^{2}\right) \rho_{1}(x)^{2} \rho_{2}(x) \\
+ & \left(A_{1}(x)-A_{1}(x) A_{2}(x)-3 A_{2}(x)-6+A_{1}(x)^{2}\right) \rho_{1}(x) \rho_{2}(x)^{2} \\
+ & \left(2 C_{1}(x)-A_{2}{ }^{\prime}(x)-2 B_{2}(x)+A_{1}(x) C_{2}(x)+C_{1}(x) A_{2}(x)\right. \\
& +12 Y(x)-2 C_{2}(x) A_{2}(x)+2 B_{1}(x) A_{1}(x)-A_{1}(x) B_{2}(x) \\
& \left.+A_{1}{ }^{\prime}(x)-A_{2}(x) B_{1}(x)\right) \rho_{1}(x) \rho_{2}(x)=0 .
\end{aligned}
$$


The dominant balances of this equation give

$$
\begin{aligned}
-A_{2}(x)+A_{1}(x) A_{2}(x)+3 A_{1}(x)+6-A_{2}(x)^{2} & =0 \\
3 B_{1}(x)-B_{2}(x)-6 Y(x)-A_{2}(x) B_{2}(x)+A_{1}(x) B_{2}(x) & =0 \\
A_{1}(x)-A_{1}(x) A_{2}(x)-3 A_{2}(x)-6+A_{1}(x)^{2} & =0 \\
C_{1}(x)-6 Y(x)-3 C_{2}(x)-C_{1}(x) A_{2}(x)+A_{1}(x) C_{1}(x) & =0
\end{aligned}
$$

The sum of Eqns (4.24) and (4.26) factors to give

$$
\left(A_{2}-A_{1}\right)\left(A_{1}+A_{2}+4\right)=0 .
$$

Substitution of the first solution $A_{2}=A_{1}$ shows that

$$
A_{2}=A_{1}=-3
$$

On the other hand, the second solution $A_{1}=-A_{2}-4$ gives

$$
A_{1}=-1, A_{2}=-3 \text { or } A_{1}=-3, A_{2}=-1 \text {. }
$$

By relabelling if necessary, we take this case to be

$$
A_{1}=-1, A_{2}=-3 .
$$

\subsubsection{Case 1: $A_{2}=A_{1}=-3$}

Consider the first case (4.28). Equations (4.25) and (4.27) give

$$
3 B_{1}=B_{2}+6 Y, \quad C_{1}=3 C_{2}+6 Y .
$$

We use these to replace $B_{1}, C_{1}$ in the transformed equation (4.23). Now the dominant terms give

$$
\begin{aligned}
3 \tau_{1}= & B_{2}{ }^{\prime}(x)+\frac{3 x}{2}+\frac{B_{2}(x)^{2}}{3}-8 B_{2}(x) Y(x)+3 Y(x)^{2} \\
& -3 C_{2}(x) B_{2}(x)-3 Y^{\prime}(x) \\
\tau_{2}= & C_{2}{ }^{\prime}(x)+\frac{x}{2}+C_{2}(x)^{2}-2 B_{2}(x) Y(x)+9 Y(x)^{2} \\
& +6 C_{2}(x) Y(x)+3 Y^{\prime}(x)-C_{2}(x) B_{2}(x)
\end{aligned}
$$

The transformed equation then becomes

$$
\begin{aligned}
& -\alpha-3 a+\left(C_{2}(x)-\frac{1}{3} B_{2}(x)\right) x-2 C_{2}(x)^{2} B_{2}(x)+2 C_{2}(x)^{3}-\frac{2}{27} B_{2}(x)^{3} \\
& +\frac{2}{3} C_{2}(x) B_{2}(x)^{2}+2 B_{2}(x)^{2} Y(x)+18 C_{2}(x)^{2} Y(x)-12 C_{2}(x) B_{2}(x) Y(x) \\
& +54 C_{2}(x) Y(x)^{2}-18 B_{2}(x) Y(x)^{2}+\frac{1}{3} B_{2}^{\prime \prime}(x)-C_{2}^{\prime \prime}(x)=0
\end{aligned}
$$


which, under the change of variables

$$
B_{2}(x)=3 C_{2}(x)+3 V(x)+9 Y(x)
$$

becomes

$$
V^{\prime \prime}(x)-2 V(x)^{3}-x V(x)-\alpha=0,
$$

which is just $\mathrm{P}_{\mathrm{II}}$ with parameter $\alpha$ (the same parameter as that in the version of $\mathrm{P}_{\mathrm{II}}$ satisfied by $y(x)$ ).

These results for the coefficients $B_{1}, C_{1}, B_{2}$, motivate the change of variables

$$
\begin{aligned}
& \rho_{1}(x)=C_{2}(x)+2 Y(x)+\sigma_{1}(x) \\
& \rho_{2}(x)=C_{2}(x)+3 Y(x)+V(x)+\sigma_{2}(x)
\end{aligned}
$$

which simplify the Riccati equations for $\rho_{1}$ and $\rho_{2}$. These become

$$
\begin{aligned}
\sigma_{1}{ }^{\prime}(x)= & \sigma_{1}(x)^{2}-3 \sigma_{1}(x) \sigma_{2}(x) \\
& -2 \sigma_{1}(x) V(x)+V^{\prime}(x)+V(x)^{2}+x / 2 \\
\sigma_{2}{ }^{\prime}(x)= & \sigma_{2}(x)^{2}-3 \sigma_{1}(x) \sigma_{2}(x) \\
& +2 \sigma_{2}(x) V(x)-V^{\prime}(x)+V(x)^{2}+x / 2
\end{aligned}
$$

We can eliminate $\sigma_{2}(x)$ from this system by solving the first equation for $\sigma_{2}(x)$ and substituting the result into the second. The result is, for $W(x)=2 \sigma_{1}(x)$,

$$
\begin{aligned}
W^{\prime \prime}(x)=\frac{2}{3} & \frac{W^{\prime}(x)^{2}}{W(x)}-\frac{1}{3}\left(2 W(x)-2 V(x)+\frac{2 V^{\prime}(x)+x+2 V(x)^{2}}{W(x)}\right) W^{\prime}(x) \\
& +\frac{2}{3} W(x)^{3}-\frac{10}{3} V(x) W(x)^{2}+\frac{1}{3}\left(6 V(x)^{2}+10 V^{\prime}(x)-x\right) W(x) \\
& +\frac{8}{3} V(x)^{3}+\frac{4}{3} x V(x)+1+\frac{8}{3} V(x) V^{\prime}(x)+2 \alpha-\frac{1}{3}\left(x^{2}+4 V(x)^{4}\right. \\
& \left.+4 x V^{\prime}(x)+8 V(x)^{2} V^{\prime}(x)+4 x V(x)^{2}+4\left(V^{\prime}(x)\right)^{2}\right) \frac{1}{W(x)}(4.34)
\end{aligned}
$$

This is $\mathrm{P}_{\mathrm{xxxy}}$ whose general form (where the functions $r(x)$ and $q(x)$ are as given in [26]) is

$$
\begin{aligned}
\frac{d^{2} W}{d x^{2}}=\frac{2}{3 W}\left(\frac{d W}{d x}\right)^{2}-\left(\frac{2 W}{3}-\frac{2 q}{3}-\frac{r}{W}\right) \frac{d W}{d x} \\
+\frac{2 W^{3}}{3}-\frac{10}{3} q W^{2}+\left(4 q^{\prime}+r+8 q^{2} / 3\right) W \\
+2 q r-3 r^{\prime}-\frac{3 r^{2}}{W} .
\end{aligned}
$$


Equation (4.34) is this equation with choices of coefficients given by

$$
q(x)=V(x), \quad r(x)=-\frac{2}{3}\left(V^{\prime}(x)+V(x)^{2}\right)-\frac{1}{3} x .
$$

(We could have eliminated $\sigma_{1}$ in the above instead of $\sigma_{2}$. The result is the same except for a sign change: $q=-V$.) Writing the Bäcklund transformation in terms of $W(x)=2 \sigma_{1}(x)$ gives

$$
\begin{aligned}
y(x) & =\sigma_{1}(x)-\sigma_{2}(x)-V(x) \\
& =\frac{1}{3} W(x)-\frac{1}{3} \frac{x}{W(x)}+\frac{1}{3} \frac{W^{\prime}(x)}{W(x)}-\frac{2}{3} \frac{V(x)^{2}}{W(x)}-\frac{1}{3} V(x)-\frac{2}{3} \frac{V^{\prime}(x)}{W(x)}
\end{aligned}
$$

with inverse

$$
W(x)=\frac{y^{\prime}(x)-V^{\prime}(x)+y(x)^{2}-V(x)^{2}}{y(x)-V(x)} .
$$

Hence we have reproduced the relation between two solutions of $\mathrm{P}_{\mathrm{II}}$ and a solution of $\mathrm{P}_{\mathrm{xxxv}}$ that has been known since classical studies of these equations [26.

\subsubsection{Case 2: $A_{1}=-1, A_{2}=-3$.}

Now we consider the second case of possible values of $A_{1}, A_{2}$ given by Eqn (4.29). Here we find from (4.25) and (4.27) that

$$
\begin{aligned}
& C_{1}(x)=C_{2}(x)+2 Y(x) \\
& B_{1}(x)=-\frac{1}{3} B_{2}(x)+2 Y(x) .
\end{aligned}
$$

Substituting these values of $C_{1}(x)$ and $B_{1}(x)$ into (4.23) gives (from the coefficient of $\left.\rho_{1}(x) \rho_{2}(x)\right)$

$$
B_{2}(x)=3 C_{2}(x)+9 Y(x) .
$$

The transformed equation then gives

$$
\begin{aligned}
& \tau_{1}(x)=C_{2}^{\prime}(x)-C_{2}(x) Y(x)-2 Y(x)^{2}+2 Y^{\prime}(x) \\
& \tau_{2}(x)=C_{2}^{\prime}(x)-2 C_{2}(x)^{2}-9 C_{2}(x) Y(x)-9 Y(x)^{2}+3 Y^{\prime}(x)+\frac{1}{2} x
\end{aligned}
$$

and substitution of these values of $\tau_{1}(x)$ and $\tau_{2}(x)$ gives

$$
\alpha=-1 / 2 \text {. }
$$

Our subsequent results apply only to this special case of $\mathrm{P}_{\mathrm{II}}$. 
Making the change of variables

$$
\begin{aligned}
& \rho_{1}(x)=C_{2}(x)+2 Y(x)+\sigma_{1}(x) \\
& \rho_{2}(x)=C_{2}(x)+3 Y(x)+\sigma_{2}(x)
\end{aligned}
$$

we find that the BT becomes

$$
y(x)=\sigma_{1}(x)-\sigma_{2}(x) .
$$

Note that $Y$ and $a$ have been eliminated here. The equations satisfied by $\sigma_{1}(x)$ and $\sigma_{2}(x)$ now yield two (apparently) different second-order equations. Elimination of $\sigma_{2}(x)$ gives

$$
\sigma_{1}^{\prime \prime}(x)-2 \sigma_{1}(x)^{3}+\frac{1}{2} x \sigma_{1}(x)=0
$$

which is equivalent to $\mathrm{P}_{\mathrm{II}}$ with zero value of the parameter. The $\mathrm{BT}$ in this case is

$$
y(x)=\frac{\sigma_{1}{ }^{\prime}(x)}{\sigma_{1}(x)}
$$

where $y(x)$ satisfies $\mathrm{P}_{\mathrm{II}}$ with $\alpha=-1 / 2$. This $\mathrm{BT}$, from $\mathrm{P}_{\mathrm{II}}$ with $\alpha=0$ to $\mathrm{P}_{\text {II }}$ with $\alpha=-1 / 2$, does not seem to be widely known, although it can in fact be found in [24].

On the other hand, elimination of $\sigma_{1}(x)$ gives (with $\sigma_{2}(x)=-W(x)$ )

$$
\begin{aligned}
W^{\prime \prime}(x)= & \frac{2}{3} \frac{\left(W^{\prime}(x)\right)^{2}}{W(x)}-\frac{1}{3}\left(2 W(x)-\frac{x}{2 W(x)}\right) W^{\prime}(x)+\frac{2}{3} W(x)^{3} \\
& +\frac{1}{6} x W(x)-\frac{1}{12} \frac{x^{2}}{W(x)}-\frac{1}{2}
\end{aligned}
$$

which can be rescaled onto $\mathrm{P}_{\mathrm{xxxv}}$ with $q=0, r=-x / 3$. The $\mathrm{BT}$ in this case is

$$
y(x)=\frac{2}{3} W(x)-\frac{W^{\prime}(x)}{3 W(x)}-\frac{x}{6 W(x)} .
$$

We now briefly consider the results obtained from our double-singularity approach if we assume our Riccati system to be

$$
\begin{aligned}
& \rho_{1}{ }^{\prime}(x)=\rho_{1}(x)^{2}+A_{1}(x) \rho_{1}(x) \rho_{2}(x)+B_{1}(x) \rho_{1}(x) \\
& +C_{1}(x) \rho_{2}(x)+\tau_{1}(x) \\
& \rho_{2}{ }^{\prime}(x)=-\rho_{2}(x)^{2}+A_{2}(x) \rho_{1}(x) \rho_{2}(x)+B_{2}(x) \rho_{1}(x) \\
& +C_{2}(x) \rho_{2}(x)+\tau_{2}(x)
\end{aligned}
$$


With this choice of Riccati system we obtain two inequivalent choices of coefficients $A_{1}(x), A_{1}(x)$. These are:

$$
A_{1}(x)=3, \quad A_{2}(x)=-3, \quad \text { or } \quad A_{2}(x)=A_{1}(x)+2 .
$$

With the first of these choices our final results are that under the change of variables

$$
\begin{aligned}
& \rho_{1}(x)=\frac{1}{3} B_{2}(x)-Y(x)+\sigma_{1}(x) \\
& \rho_{2}(x)=\frac{1}{3} B_{2}(x)+\sigma_{2}(x)
\end{aligned}
$$

our Bäcklund transformation becomes

$$
y(x)=\sigma_{1}(x)-\sigma_{2}(x),
$$

where $\sigma_{1}(x)$ and $\sigma_{2}(x)$ satisfy the Riccati system

$$
\begin{aligned}
& \sigma_{1}^{\prime}(x)=\sigma_{1}(x)^{2}+3 \sigma_{1}(x) \sigma_{2}(x)-\frac{1}{4} x \\
& \sigma_{2}^{\prime}(x)=-\sigma_{2}(x)^{2}-3 \sigma_{1}(x) \sigma_{2}(x)+\frac{1}{4} x
\end{aligned}
$$

and we have in addition the compatibility condition (resulting from the transformed equation)

$$
\alpha=-\frac{1}{2}
$$

Note that again $Y$ and $a$ play no role in our final results. Eliminating $\sigma_{2}(x)$ from the above system and then substituting $\sigma_{1}(x)=W(x) / 2$ yields equation (4.35), together with the same Bäcklund transformation (4.36). On the other hand, eliminating $\sigma_{1}(x)$ from the above Riccati system yields (4.35) in $W(x)=-2 \sigma_{2}(x)$.

With the second choice of coefficients $A_{i}(x)$ we find only that $y(x)=u(x)$ satisfies $\mathrm{P}_{\mathrm{II}}$ for $\alpha=1 / 2$, where $u(x)=\rho_{1}(x)-\rho_{2}(x)+Y(x)$ is a solution of

$$
u^{\prime}(x)=u(x)^{2}+\frac{1}{2} x
$$

i.e. we find only the special integral of $\mathrm{P}_{\mathrm{II}}$ which gives rise to Airy function solutions [24]. 


\section{$4.2 \quad \mathrm{P}_{\mathrm{IV}}$}

Dominant balances of the transformed $\mathrm{P}_{\mathrm{IV}}$ equation yield only one consistent choice of $A_{1}, A_{2}$, namely

$$
A_{1}(x)=A_{2}(x)=-2 .
$$

The resulting equation shows that

$C_{2}(x)=-2 x-2 Y(x), \quad B_{1}(x)=2 x+2 Y(x), \quad C_{1}(x)=-4 x-2 Y(x)+B_{2}(x)$,

and that

$$
\begin{aligned}
\tau_{1}(x)= & \frac{1}{2} B_{2}^{\prime}(x)-\frac{1}{4} B_{2}(x)^{2}+x B_{2}(x)-Y^{\prime}(x)+Y(x)^{2}+2 x Y(x) \\
& -\alpha-1 \pm \frac{1}{2} \gamma \\
\tau_{2}(x)= & \frac{1}{2} B_{2}^{\prime}(x)-\frac{1}{4} B_{2}(x)^{2}+x B_{2}(x)+Y(x) B_{2}(x)-\alpha-1 \mp \frac{1}{2} \gamma
\end{aligned}
$$

Defining $\rho_{i}$ now by taking

$$
\begin{aligned}
\rho_{1}(x) & =\frac{1}{2} B_{2}(x)-Y(x)-2 x+\sigma_{1}(x) \\
\rho_{2}(x) & =\frac{1}{2} B_{2}(x)+\sigma_{2}(x),
\end{aligned}
$$

the BT becomes

$$
y(x)=\sigma_{1}(x)-\sigma_{2}(x)-2 x .
$$

Once again the intermediate variable $Y(x)$ (and the parameters $a$ and $c$ ) has been eliminated and we are now transforming to $y(x)$ from $\sigma_{1}$ or $\sigma_{2}$.

Eliminating $\sigma_{2}(x)$ leads to a different version of $\mathrm{P}_{\mathrm{IV}}$

$$
\sigma_{1}^{\prime \prime}(x)=\frac{\sigma_{1}{ }^{\prime}(x)^{2}}{2 \sigma_{1}(x)}+\frac{3}{2} \sigma_{1}(x)^{3}-4 x \sigma_{1}(x)^{2}+2\left(x^{2}-A\right) \sigma_{1}(x)-\frac{C^{2}}{2 \sigma_{1}(x)}
$$

where

$$
\begin{aligned}
A & =-\frac{1}{2} \alpha \mp \frac{3}{4} \gamma-\frac{1}{2} \\
C^{2} & =\frac{1}{4}( \pm \gamma-2 \alpha+2)^{2}
\end{aligned}
$$

(This is the conventional form of $\mathrm{P}_{\mathrm{IV}}$ if we take $V=-\sigma_{1}$.) In this case, the BT becomes

$$
y(x)=\frac{\sigma_{1}{ }^{\prime}(x)+\sigma_{1}(x)^{2}-2 x \sigma_{1}(x)-(1-\alpha \pm \gamma / 2)}{2 \sigma_{1}(x)} .
$$


Elimination of $\sigma_{1}(x)$ leads to a conventional version of $\mathrm{P}_{\mathrm{IV}}$ governing $\sigma_{2}(x)$ but with new $A$ and $C$ given by

$$
\begin{aligned}
A & =-\frac{1}{2} \alpha \pm \frac{3}{4} \gamma+\frac{1}{2} \\
C^{2} & =\frac{1}{4}( \pm \gamma+2 \alpha+2)^{2}
\end{aligned}
$$

In this case, the $\mathrm{BT}$ is

$$
y(x)=-\frac{\sigma_{2}{ }^{\prime}(x)+\sigma_{2}(x)^{2}+2 x \sigma_{2}(x)+(1+\alpha \pm \gamma / 2)}{2 \sigma_{2}(x)} .
$$

Thus once again we recover the BTs $\widetilde{y}$ and $\hat{y}$ obtained in Section 3. Considering also the BT from $\sigma_{2}$ to $\sigma_{1}$ (respectively $\sigma_{1}$ to $\sigma_{2}$ ) then gives, for suitable choices of signs, $\hat{y}=\widetilde{y} \circ \widetilde{y}$ (respectively $\widetilde{y}=\hat{y} \circ \hat{y}$ ) [25].

For $\mathrm{P}_{\mathrm{IV}}$, the Riccati system (4.37), (4.38) gives only restricted results. We get that $y(x)=u(x)$ is a solution of $\mathrm{P}_{\mathrm{IV}}$ with parameters subject to the constraint $\gamma^{2}=4(\alpha+1)^{2}$, where $u(x)=\rho_{1}(x)-\rho_{2}(x)+Y(x)$ satisfies

$$
u^{\prime}(x)=u(x)^{2}+2 x u(x)-2(\alpha+1),
$$

i.e. we find only a special integral of $\mathrm{P}_{\mathrm{IV}}$.

\section{Conclusions}

We have introduced a new, general method of constructing Bäcklund transformations for ordinary differential equations. This is based on mappings preserving natural subsets of movable poles. For the examples considered here this method has allowed us to construct all known fundamental Bäcklund transformations, including a less well known Bäcklund transformation for $\mathrm{P}_{\mathrm{II}}$. Our approach has also allowed us to find Bäcklund transformations onto other ODEs in the Painlevé classification, as well as to deduce relationships between the Bäcklund transformations constructed. Its application to other Painlevé equations is discussed in [23].

\section{Acknowledgements}

Andrew Pickering and Pilar R. Gordoa are grateful to Nalini Joshi for her invitations to visit the University of Adelaide. The research in this paper was supported by the Australian Research Council. 


\section{References}

[1] M. J. Ablowitz and P. A. Clarkson. Solitons, Nonlinear Evolution Equations and Inverse Scattering, volume 149 of London Mathematical Society Lecture Notes in Mathematics. Cambridge University Press, Cambridge, 1991.

[2] M. J. Ablowitz, A. Ramani, and H. Segur. A connection between nonlinear evolution equations and ordinary differential equations of P-type I and II. J. Math. Phys., 21:715-721, 1006-1015, 1980.

[3] M. D. Kruskal, N. Joshi, and R. Halburd. Analytic and asymptotic methods for nonlinear singularity analysis: a review and extensions of tests for the Painlevé property. In Integrability of Nonlinear Systems, volume 495 of Lecture Notes in Physics, pages 171-205, Heidelberg, 1997. Springer-Verlag.

[4] M. D. Kruskal and P. A. Clarkson. The Painlevé-Kowalevski and polyPainlevé tests for integrability. Stud. Appl. Math., 86:87-165, 1992.

[5] R. Conte, A. P. Fordy, and A. Pickering. A perturbative Painlevé approach to nonlinear differential equations. Physica, 69D:33-58, 1993.

[6] J. Weiss. The Painlevé property for partial differential equations II: Bäcklund transformation, Lax pairs, and the Schwarzian derivative. J. Math. Phys., 24:1405-1413, 1983.

[7] J. Weiss, M. Tabor, and G. Carnevale. The Painlevé property for partial differential equations. J. Math. Phys., 24:522-526, 1983.

[8] N. Joshi and J. A. Petersen. A method of proving the convergence of the Painlevé expansions of partial differential equations. Nonlinearity, 7:595-602, 1994.

[9] N. Joshi and G. K. Srinivasan. The radius of convergence and wellposedness of the Painlevé expansions of the Korteweg-deVries equation. Nonlinearity, 10:71-79, 1997.

[10] P. G. Estévez, P. R. Gordoa, L. Martínez Alonso, and E. M. Reus. Modified singular manifold expansion: application to the Boussinesq and Mikhailov-Shabat systems. J. Phys. A: Math. and General, 26:19151925, 1993.

[11] P. R. Gordoa and P. G. Estévez. Double singular manifold method for the MKDV equation. Teor. Matem. Fizika, 99:370-376, 1994. 
[12] M. Musette and R. Conte. The two-singular manifold method: I modified $\mathrm{KdV}$ and sine-Gordon equations. J.Phys. A: Math and General, 27:3895-3913, 1994.

[13] R. Conte, M. Musette, and A. Pickering. The two-singular manifold method: II classical Boussinesq system. J.Phys. A: Math and General, 28:179-187, 1995.

[14] A. Pickering. The singular manifold method revisited. J. Math. Phys., 37:1894-1927, 1996.

[15] P. G. Estévez and P. R. Gordoa. Darboux transformations via Painlevé analysis. Inverse Problems, 13:939-957, 1997.

[16] J. Weiss. Bäcklund transformation and linearizations of the HénonHeiles system. Phys. Lett. A, 102:329-331, 1984.

[17] J. Weiss. Bäcklund transformation and the Hénon-Heiles system. Phys. Lett. A, 105:387-389, 1984.

[18] A. C. Newell, M. Tabor, and Y. B. Zeng. A unified approach to Painlevé expansions. Physica D, 29:1-68, 1987.

[19] J. D. Gibbon, A. C. Newell, M. Tabor, and Y. B. Zeng. Lax pairs, Bäcklund transformations and special solutions for ordinary differential equations. Nonlinearity, 1:481-490, 1988.

[20] P. A. Clarkson, Nalini Joshi, and Andrew Pickering. Bäcklund transformations for the second Painlevé hierarchy: a modified truncation approach. Inverse Problems, 15: 175-187, 1999.

[21] N. Joshi and A. Pickering. Bäcklund transformations for similarity reductions of the modified Sawada-Kotera / Kaup-Kupershmidt hierarchy, submitted, 1999.

[22] N. Joshi and M. D. Kruskal. A direct proof that the solutions of the six Painlevé equations have no movable singularities except poles. Stud. Appl. Math., 93:187-207, 1994.

[23] P. R. Gordoa, N. Joshi and A. Pickering, Mappings preserving locations of movable poles II, in preparation, 1999.

[24] B. Gambier. Sur les equations differentielles du second ordre et du premier degré dont l'intégrale générale est à points critiques fixes. Acta Math., 33:1-55, 1910. 
[25] A. Bassom, P. A. Clarkson, and A. C. Hicks. Bäcklund transformations and hierarchies of exact solutions for the fourth Painlevé equation. Stud. Appl. Math., 95:1-71, 1995.

[26] E. L. Ince. Ordinary Differential Equations. Dover, New York, 1954. 